\section{Abscess formation in a giant gastrointestinal stromal tumor of the stomach following endoscopic biopsy}

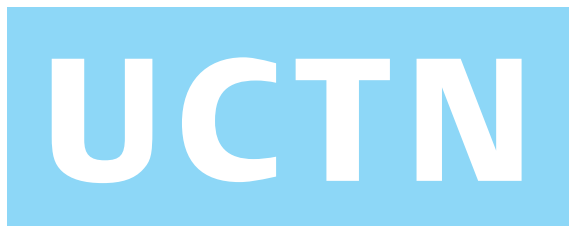

The development of transient bacteremia during or after gastrointestinal endoscopy is a well-known phenomenon but one which usually has no clinical significance. Antibiotic prophylaxis is not recommended, except for patients at high risk [1]. However, we had a patient with a gastric gastrointestinal stromal tumor who developed an abscess after undergoing endoscopic biopsy.

A 74-year-old man with severe anemia underwent gastrointestinal endoscopy, which revealed an area of ulceration, $2.5 \mathrm{~cm}$ in diameter, on the anterior wall of the upper gastric body (Figure 1). Abdominal computed tomography showed a solid extraluminal gastric tumor that extended to the left subphrenic space. Biopsies were taken from the bottom of the ulcer using standard biopsy forceps. After this procedure, the patient developed sepsis and the anaerobic bacterium, Clostridium perfringens was isolated from his blood cultures. Computed tomography was repeated on day 7 after the endoscopic biopsy and this revealed a closed abscess with a fluid level in the extraluminal gastric tumor (Figure 2).

Emergency surgery (proximal partial gastrectomy) was performed to excise the tumor and abscess. The tumor was on the anterior wall of the stomach, extending from the fornix to the upper body. The excised submucosal tumor was $12 \mathrm{~cm} \times 13 \mathrm{~cm}$ in diameter, with an overlying ulcer on the gastric mucosa $(2.5 \mathrm{~cm} \times 1.5 \mathrm{~cm})$, but with no obvious fistula between the gastric lumen and the tumor cavity. It was diagnosed as a gastrointestinal stromal tumor on the basis of the histological and immuohistochemical findings, including the identificaton of spindle cells with a storiform growth pattern, and positive staining for KIT (CD117) [2].

It was thought there was no permanent bacterial flora in the gastric lumen because of the low $\mathrm{pH}$ in this part of the gastrointestinal tract. However, it has been

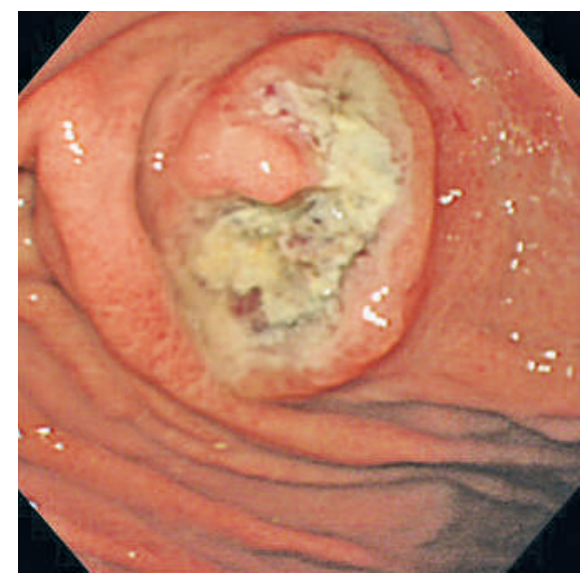

Figure 1 Gastroscopy performed on the day of admission showed an area of ulceration, $2.5 \mathrm{~cm}$ in diameter, on the anterior wall of the upper gastric body.

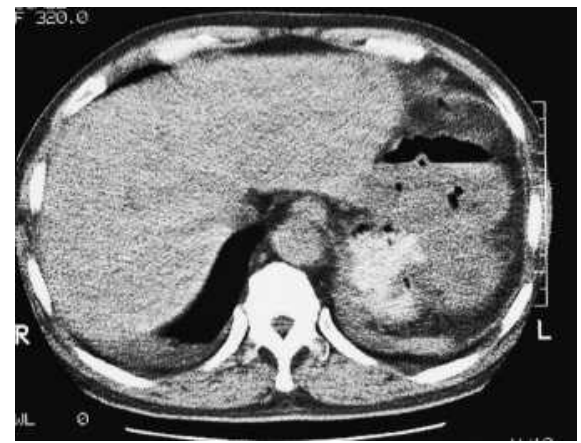

Figure 2 Abdominal computed tomography on day 7 after the endoscopic biopsy revealed a fluid level in the extraluminal gastric tumor, which extended from the upper gastric body into the left subphrenic space. Orally administered contrast medium was observed in the stomach but did not enter the tumor cavity, and it was concluded that this was a closed abscess in the tumor.

suggested that bacterial pathogens can come from the oropharyngeal flora [3]. In addition, bacterial overgrowth, including overgrowth with anaerobic bacteria, has been observed in the stomach in patients taking drugs that reduce the secretion of gastric acid, particularly the protonpump inhibitors [4].

In conclusion, notwithstanding the location, endoscopic biopsies from an ulcer overlying a large submucosal tumor in the upper gastrointestinal tract should be taken carefully because of the potential risk of infection.

\section{Endoscopy_UCTN_Code_CPL_1AH_2AB}

\section{S. Nozawa, T. Bando, T. Nagata,}

\section{K. Tsukada}

The Second Department of Surgery, Toyama Medical and Pharmaceutical University, Toyama, Japan.

\section{References}

${ }^{1}$ American Society for Gastrointestinal Endoscopy. Antibiotic prophylaxis for gastrointestinal endoscopy. American Society for Gastrointestinal Endoscopy. Gastrointest Endosc 1995; 42: 630-635

2 Miettinen M, Sobin LH, Lasota J. Gastrointestinal stromal tumors of the stomach: a clinicopathologic, immunohistochemical, and molecular genetic study of 1765 cases with long-term follow-up. Am J Surg Pathol 2005; 29: $52-68$

${ }^{3}$ Schlaeffer F, Riesenberg K, Mikolich Det al. Serious bacterial infections after endoscopic procedures. Arch Intern Med 1996; 156: 572-574

${ }^{4}$ Thorens J, Froehlich F, Schwizer Wet al. Bacterial overgrowth during treatment with omeprazole compared with cimetidine: a prospective randomised double blind study. Gut 1996; 39: 54-59

\section{Corresponding author}

\section{S. Nozawa, M.D.}

Department of General Surgery

Graduate School of Medicine

Chiba University

1-8-1 Inohana

Chuou-ku

Chiba 260-8670

Japan

Fax: $\quad+81-43-226-2552$

E-mail: snozawa-gi@umin.ac.jp 